\title{
Prospective study on radiation dose escalation in the treatment of squamous cell carcinoma of oesophagus with definitive concurrent chemo-radiation using three-dimensional conformal radiation therapy
}

\author{
Ashwini L. ${ }^{1}$, Kumar Kn P. ${ }^{2 *}$, Pavithran K. ${ }^{3}$, Ragavendra H. ${ }^{4}$ \\ DOI: https://doi.org/10.17511/ijmrr.2019.i05.02
}

${ }^{1}$ Ashwini L., Radiation Oncologist, Department of Radiation Oncology, Medical Physicist-Amrita Institute of Medical Sciences, Kochi, Kerala, India.

2* Pradeep Kumar Kn, Radiation Oncologist, Department of Radiation Oncology, Srinivasam Cancer Care Multispeciality Hospital, Bangalore, Karnataka, India.

3 Pavithran K., Medical Oncologist, Medical Physicist-Amrita Institute of Medical Sciences, Kochi, Kerala, India.

${ }^{4}$ Ragavendra H., Medical Physicist-Amrita Institute of Medical Sciences, Kochi, Kerala, India.

Background: Poor local control and survival rates associated with radiation and chemotherapy in the management of locally advanced carcinoma of oesophagus have encouraged use of radiation dose escalation. This study analysed outcome and tolerance of radiation dose escalation with concurrent chemotherapy in non-metastatic squamous cell carcinoma of oesophagus. Materials and Methods: Patients diagnosed with stage I-III squamous cell carcinoma oesophagus, intended to be treated with radical chemo-radiation, between August 2015 to August 2017 were included in this prospective study. Baseline endoscopy, oral intake score and FACT E-QOL score were documented. Results: Out of total of 20 eligible patients with median age of 64 years, majority had middle thoracic oesophageal tumour $(60 \%)$, grade 2 dysphagia $(75 \%)$ on oral intake scale. Patients with tumour length of $\leq 5 \mathrm{~cm}(55 \%)$ or $>5 \mathrm{~cm}(45 \%)$ were categorised. Majority patients $(85 \%)$ showed complete response at 3 months post treatment. The oral intake score and quality of life improved in $55 \%$ and $85 \%$ of the patients, respectively. Ten patients had disease progression with $40 \%$ of patients showing disease recurrence. Conclusion: Radiation dose escalation with concurrent chemotherapy in the management of stage I-III, non-metastatic squamous cell carcinoma oesophagus yields a good local control rate at 3 months with less severe complications and improved quality of life.

Keywords: Radiation dose, Squamous cell carcinoma, Oesophagus, Chemo-radiation

Corresponding Author

Pradeep Kumar Kn, Radiation Oncologist, Department of Radiation Oncology, Srinivasam Cancer Care Multispeciality Hospital, Bangalore, Karnataka, India. Email: pradeepkrn99@gmail.com
How to Cite this Article

Ashwini L, Kumar Kn P, Pavithran K, Ragavendra H. Prospective study on radiation dose escalation in the treatment of squamous cell carcinoma of oesophagus with definitive concurrent chemo-radiation using three-dimensional conformal radiation therapy. Int ] Med Res Rev. 2019;7(5):348-356.

Available From

https://ijmrr.medresearch.in/index.php/ijmrr/article/ view/1080

\begin{tabular}{|c|c|}
\hline $\begin{array}{c}\text { Manuscript Received } \\
2019-07-30\end{array}$ & $\begin{array}{c}\text { Review Round } 1 \\
2019-08-10\end{array}$ \\
\hline $\begin{array}{c}\text { Conflict of Interest } \\
\text { No }\end{array}$ & $\begin{array}{c}\text { Funding } \\
\mathrm{Nil}\end{array}$ \\
\hline
\end{tabular}

To Browse

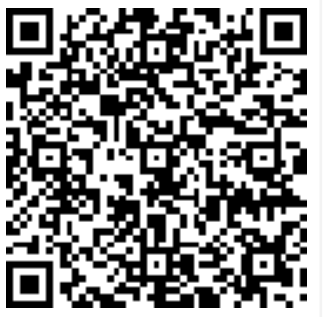




\section{Introduction}

Emerging trends suggest that the prevalence of carcinoma oesophagus has increased owing to lifestyle and other factors. According to Globocan 2018 report, worldwide cancer statistics indicate that there are $5,72,034(3.2 \%)$ cases of oesophagus cancer per year, resulting in approximately $5,08,585(5.3 \%)$ deaths per year. In India, estimated number of new cases diagnosed with oesophagus cancer were 4,44,597 (5.1\%) [1]. Being the sixth most common cancer among men in India, it has been known to have poor prognosis due to delayed presentation and rapid progression.

Over the past two decades, there have been many significant changes in the management of oesophageal cancer. Early stage, superficial oesophageal cancers can be manged with surgery alone and can provide good disease-free survival times. However, late stage carcinomas are difficult to manage with surgery alone, due to high risk of local-regional failure and progression to distant metastases. The challenges and poor survival outcomes involving surgical intervention alone, has prompted considerable research on multimodality treatment options.

The morbidity and mortality observed after neoadjuvant chemo-radiotherapy followed by surgery, prompted the use chemo-radiation as standard of care, in locally advanced cases or in patients with poor performance status [24]. National Comprehensive Cancer Network (NCCN)oesophageal cancer guidelines recommend preoperative CCRT or definitive CCRT for the management of patients with Stage II or III oesophageal cancer [5].

The advances in radiation therapy have encouraged the use of dose escalation, in order to achieve good local control thereby, improving the progression free survival and overall survival, which is equivalent to chemo-radiotherapy followed by surgery. Following the INT01-23 trial which reported increased treatment-related deaths with dose escalation, multiple retrospective, phase II and phase III randomized controlled studies have proven the efficacy of radiation dose escalation [69].

However, the implementation of varying schedules of radiation and chemotherapy regimen has led to difference in the results. A recent a systematic review and pooled analysis, which evaluated benefits of high-dose ( $\geq 60 \mathrm{~Gy}$ ) radiotherapy in
Definitive concurrent chemoradiotherapy compared to conventional-dose concurrent chemoradiotherapy, concluded that $\geq 60$ Gy concurrent chemoradiotherapy improved clinical outcomes compared to the conventional-dose concurrent chemoradiotherapy [10].

There is no data studying the effect of radiation dose escalation with concurrent chemotherapy, using external beam radiation therapy in Indian population. The present study made an attempt to analyse the local control, dysphagia status and quality of life following the dose escalation treatment with concurrent chemotherapy in squamous cell oesophageal carcinoma.

\section{Materials and Methods}

Study design, duration and setting: A prospective study was conducted in the Department of Radiation Oncology, Srinivasam Cancer Care Multispeciality Hospital, Bangalore, Karnataka, during August 2015 to August 2017.

Ethical considerations: The study protocol was approved by Institutional Ethics Committee and conducted in accordance with the principles of Declaration of Helsinki. Written informed consent was obtained from all patients before enrolment into the study.

Inclusion and exclusion criteria: The study included patients with stage I-III squamous cell carcinoma of oesophagus who were intended to treat radically with chemo-radiation between August 2015 to August, 2017. Patients with tumour in the cervical oesophagus, gastro-oesophageal junction and distant metastases were excluded.

Treatment and data collection: All patients were administered with total radiation dose of 60Gy (2Gy per fraction) and concurrent 3 weekly 5-FU/Cisplatin or weekly Cisplatin chemotherapy. All patients were treated with radiation therapy using threedimensional conformal radiotherapy on a linear accelerator. The initial phase of EBRT was planned for the gross tumour volume with a $3 \mathrm{~cm}$ margin cranio-caudal and $1 \mathrm{~cm}$ axial margin, to a dose of 46Gy (2Gy per fraction) followed by boost phase including the gross tumour volume alone to a dose of 14Gy (2Gy per fraction) using antero-posterior or oblique fields to achieve dose constraints.

All patients received concurrent chemotherapy with either 3-weekly 5-FU/Cisplatin regimen or weekly Cisplatin alone, during the course of radiation. 
Patients were evaluated once a week for radiation and chemotherapy- related toxicity. If any patient developed Grade 3 or higher toxicity, treatment break was given; and resumed after clinical improvement. A baseline oral intake score and Functional Assessment of Cancer Therapy Esophagus Cancer (FACT E) score for quality of life were also recorded.

Response assessment: Patients were evaluated with oesophago-gastro-duodenal (OGD) scopy at 3 months after completion of treatment to assess local response, oral intake score for dysphagia and FACT E score for quality of life. The parameters were compared with baseline scores to identify improvement or worsening. Patients were further followed up till the time of analysis, for recurrence or disease progression.

The disease-free survival was defined as the time from the date of diagnosis to that of disease progression censored to last date of follow up. Overall survival was defined as the time from the date of diagnosis till death or censored to last date of follow up.

Statistical analysis: All continuous parameters were measured as median (minimum to maximum). Statistical analysis was done using IBM SPSS 20. (SPSS Inc.; Chicago, IL, USA). Kaplan Meir survival curves were obtained. For all the categorical variables, the results are given as percentage. To obtain the association of categorical variables, chi square test was applied. The $p<0.05$ was considered as statistically significant.

\section{Results}

A total number of 20 patients newly diagnosed with stage I-III squamous cell carcinoma of thoracic oesophagus and who were intended to radically treat with radiation dose escalation and concurrent chemotherapy were prospectively analysed.

Patients were between the age group of 24 years to 74 years with median age of 64 years. The Karnofsky's performance status of most of the patients was above 80. At presentation, majority had Grade 2 dysphagia according to oral intake scale. Two-thirds of the population in the study had tumour in the middle thoracic oesophagus (Table 1 ).

Table-1: Patient characteristics.

\begin{tabular}{|c|l|}
\hline \multicolumn{1}{|c|}{ Characteristics } & \multicolumn{1}{c|}{ Value } \\
\hline Median age (years) & $64(34-74)$ \\
\hline
\end{tabular}

\begin{tabular}{|c|c|}
\hline \multicolumn{2}{|c|}{ Karnofsky's performance score: } \\
\hline$>80$ & 12 \\
\hline $60-80$ & 8 \\
\hline \multicolumn{2}{|c|}{ Oral intake score at presentation: } \\
\hline Grade 1 (normal) & 0 \\
\hline Grade 2 (solids) & $12(60)$ \\
\hline Grade 3 (semi-solids) & $6(30)$ \\
\hline Grade 4 (liquids) & $2(10)$ \\
\hline \multicolumn{2}{|c|}{ Location of tumour on OGD scopy: } \\
\hline Upper thoracic $(18-24 \mathrm{~cm})$ & $2(10)$ \\
\hline Middle thoracic $(24-32 \mathrm{~cm})$ & $15(75)$ \\
\hline Lower thoracic $(32-38 \mathrm{~cm})$ & $3(15)$ \\
\hline \multicolumn{2}{|c|}{ Tumour volume along the lumen: } \\
\hline$\leq 5 \mathrm{~cm}$ & $11(55)$ \\
\hline$>5 \mathrm{~cm}$ & $9(45)$ \\
\hline \multicolumn{2}{|l|}{ Stage (AJCC 7th edition): } \\
\hline I & 0 \\
\hline IIA & $5(25)$ \\
\hline IIB & $4(20)$ \\
\hline IIIA & $7(35)$ \\
\hline IIIB & 0 \\
\hline IIIC & $4(20)$ \\
\hline \multicolumn{2}{|l|}{ Chemotherapy: } \\
\hline 5-FU \&Cisplatin regimen & $13(65)$ \\
\hline Weekly Cisplatin & 7 (35) \\
\hline
\end{tabular}

Data presented as $\mathrm{n}(\%)$, unless otherwise specified. AJCC, American Joint Committee on Cancer; OGD, oesophago-gastro-duodenal.

Dose-volume parameters: The mean dose to the combined lung volume (Dmean_comblung), volume of right and left receiving 20Gy (V20_rt_lung and V20_It_lung, respectively), mean dose received by the heart (Dmean_heart) and maximum dose received by the heart (Dmax_heart) were documented. The median of each parameter was calculated. (Table 2)

Table-2: Dose volume parameters.

\begin{tabular}{|l|l|l|}
\hline \multicolumn{1}{|c|}{ Parameters } & \multicolumn{1}{c|}{ Median } & \multicolumn{1}{c|}{ Range } \\
\hline Dmean_comblung(cGy) & 1181 & $471-1459$ \\
\hline V20_rt_lung (\%) & 18.81 & $4.02-29.59$ \\
\hline V20_It_lung (\%) & 16.05 & $1.25-30.91$ \\
\hline Dmean_heart(cGy) & 1960 & $200-3560$ \\
\hline Dmax_heart(cGy) & 6002 & $350-6230$ \\
\hline
\end{tabular}

Local response, dysphagia, toxicity and quality of life at $\mathbf{3}$ months: Using the tools for assessing the tumour response, dysphagia and quality of life, patients were categorised into complete response versus failure, improvement versus worsening of oral intake score and FACT-E score (Table 3). 
One patient in this study with a background of Fanconi's anaemia developed acute cardiac event within 3 months after completion of radiation therapy. She was treated with conservative measures.

Table-3: Representing the assessment, 3 months post treatment.

\begin{tabular}{|l|l|}
\hline \multicolumn{1}{|c|}{ Assessment tools } & \multicolumn{1}{c|}{ n (\%) } \\
\hline Tumour response on OGD scopy: & $17(85)$ \\
\hline Complete response & $1(5)$ \\
\hline Residue & $2((10)$ \\
\hline Failure & $11(55)$ \\
\hline Oral intake score: & $9(45)$ \\
\hline Improvement & \multicolumn{2}{|l}{} \\
\hline Worsening & $15(75)$ \\
\hline RTOG toxicity criteria for oesophagus: & $5(15)$ \\
\hline Grade 2 or less & $17(85)$ \\
\hline$>$ Grade 2 & $3(15)$ \\
\hline FACT E score for quality of life: & \multicolumn{2}{|l}{} \\
\hline Improvement &
\end{tabular}

FACT E, Functional Assessment of Cancer Therapy Esophagus Cancer; OGD, oesophago-gastroduodenal; RTOG, Radiation Therapy Oncology Group. The primary objective of the study was to assess the tumour response, 3 months after completion of radiation treatment. Eighty-five percent of the patients had complete disappearance of the tumour, one patient had residue and two patients had progressive disease in the oesophagus. With respect to FACT E questionnaire, $85 \%$ of the patients had improvement in the quality of life post treatment at 3 months.

Fifteen percent of the patients had more than Grade 2 toxicity as per Radiation Therapy Oncology Group (RTOG) toxicity criteria. The patient who had residual disease and two patients with Grade 3 toxicity underwent self-expandable metallic stent (SEMS) placement; and overall five patients from the entire study required OGD scopy guided dilatation of the oesophageal stricture and four patients who had Grade 2 toxicity were treated with steroids for symptom relief. Two patients with poor oral intake and significant weight loss had Ryle's tube insertion to maintain nourishment.

The Grades of dysphagia at 3 months, based on RTOG acute toxicity criteria was compared to find an association with tumour volume $(\leq 5 \mathrm{~cm}$ or $>5 \mathrm{~cm}$ ) using Chi-square test (Table 4, Figure7). No statistical significance was found $(p=0.157)$.
Follow up: All patients were followed up two monthly, after the first three months follow-up, unless symptomatic. The median (range) follow-up period of the study was 14.5 months (4-30months). During the follow-up, patients were evaluated for toxicity, loco-regional and distant failure.

Toxicity: Two patients, who had persistent stricture (Grade 3 toxicity) despite repeated dilatation, underwent SEMS placement. Hence, a total of three patients out of 20 required SEMS placement in view of Grade 3 toxicity post radiation. Rest of them were relatively symptom free and were managed conservatively.

Pattern of failure: A total of 10 patients (50\%) among 20, failed either loco-regionally or distally during the follow-up. With a median disease-free survival of 16 months (95\% Confidence interval [CI]: 10-21months), the disease-free survival rates at 12 months and 16 months were $62.9 \%$ and $45.9 \%$, respectively [Table 4 ].

Table-4: Pattern of failure.

\begin{tabular}{|l|l|}
\hline \multicolumn{1}{|c|}{ Disease status } & \multicolumn{1}{|c|}{$\mathbf{n}(\%)$} \\
\hline Disease free & $10(50)$ \\
\hline Failure: & $3(15)$ \\
\hline Loco-regional and Distant failure & $7(35)$ \\
\hline Distant failure alone &
\end{tabular}

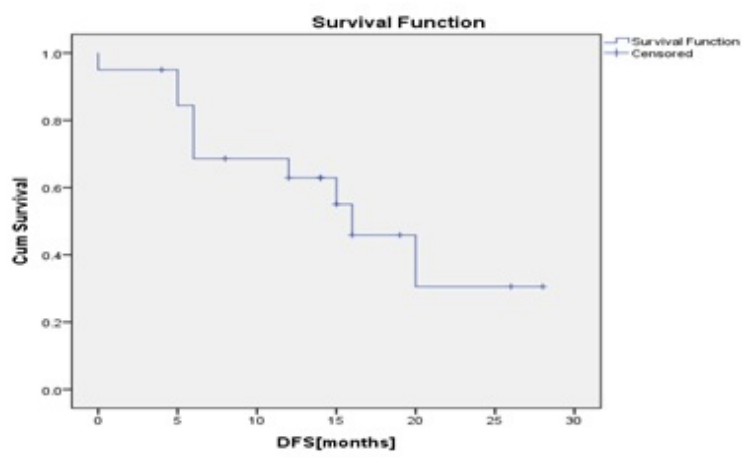

Figure-1: Kaplan Meir curve representing the disease-free survival

Survival: Out of the 20 patients, 12 patients were alive, and eight patients died (Table 5). Among the patients who are alive, two had disease progression and remaining ten patients were without any evidence of recurrence or progression. (Figure 2)

Table-5: Survival status.

\begin{tabular}{|l|l|l|}
\hline \multicolumn{1}{|c|}{ Status } & Frequency & \multicolumn{1}{c|}{ Percentage (\%) } \\
\hline Alive without disease & 10 & $50 \%$ \\
\hline Alive with disease & 2 & $10 \%$ \\
\hline Dead & 8 & $40 \%$ \\
\hline
\end{tabular}




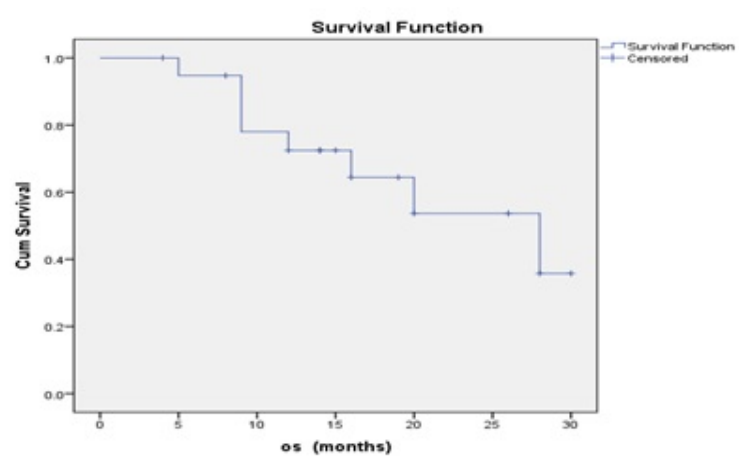

Figure-2: Kaplan Meir curve representing the overall survival

Salvage treatment: On follow up, nine patients out of 20 progressed either locally or distally. Salvage treatment in the form of radiation to the node recurrence, lung metastases, bone metastases or chemotherapy were implemented after complete evaluation. Palliative care alone at the time of progression was offered to five patients.

\section{Discussion}

Treatment outcomes of carcinoma oesophagus continue to be poorer. In an attempt to improve results, various techniques have been tried, including the increasing dose of radiation, performing curative intent surgery after chemoradiation therapy, addition of intra-luminal brachytherapy, etc. Although the NCCN oesophageal cancer guidelines recommend radiation doses of $50.4 \mathrm{~Gy}$ to $54 \mathrm{~Gy}$ for definitive chemo-radiation, dose escalation should further be assessed in phase III clinical trials for better outcomes with regards to local control and overall survival.

Since the INT01-23 trial [6], there have been multiple trials attempting to prove the benefits of dose escalation in treating oesophageal carcinoma. Though the treatment related deaths in this trial were more in high dose arms, closer review showed that most of the patients died before reaching a dose of 50.4Gy. Hence, the increased death rates in high dose arm remain unexplained. A meta-analysis on the pathological complete response ( $P C R$ ) rates following higher dose of radiation suggested that higher $\mathrm{pCR}$ rates were achieved with high dose radiation [11]. However, only three of the 26 studies in this data used doses higher than 50Gy. This was further proven by Zhang et al study [12], which showed dose higher than 54Gy with concurrent chemotherapy yielded significantly better local control, disease free survival and overall survival.
A phase II study by Japan Clinical Oncology Group (JCOG 9906) [4], evaluated the efficacy of definitive concurrent chemo-radiation with 5-FU and Cisplatin with radiation dose of $60 \mathrm{~Gy}$ in 30 fractions. A twoweek break was provided after $30 \mathrm{GY}$ irradiation and radiation was resumed on 36th day. The CTV for $60 \mathrm{~Gy}$ included primary tumour with $5 \mathrm{~cm}$ craniocaudal margin and $1 \mathrm{~cm}$ axially, a PTV of $2 \mathrm{~mm}$ was given. The primary objective was to assess the overall survival; secondary endpoints were CR rates, PFS, acute \& late adverse events.

Among the 74 eligible patients, 49 deaths were reported in the final analysis. $62.2 \%$ of the patients achieved complete response. The median survival was 2.4 years, with 3 -year and 5 -year survival rates of $44.7 \%$ and $36.8 \%$. The study demonstrated a moderately high late toxicity rates with respect to pneumonitis, pleural effusion, pericarditis. The proportion of Grade 3/4 toxicity was $30.1 \%$ after 5 years from the initiation of chemo-radiation. The study insisted on the importance of reducing the volume of radiation and the use of IntensityModulated Radiation therapy (IMRT), in order to counter the late toxicity, as opposed to INT01-23 trial.

In the present study, the outcome of radiation dose escalation with concurrent chemotherapy was analysed in patients with stage I-III oesophageal squamous cell carcinoma, who were unfit or refused surgery. The patient characteristics were similar to the retrospective JCOG 9906 study with respect to age, gender, performance status, location of the tumour and stage of the tumour [13].

When compared to JCOG study in which the CTV for initial phase included the entire oesophagus and boost was treated with $5 \mathrm{~cm}$, margin to the tumour cranio-caudally and $2 \mathrm{~cm}$ axially; it gave a CTV margin of $3 \mathrm{~cm}$ cranio-caudally and $1 \mathrm{~cm}$ axially was given to the tumour which was treated with initial $46 G y$ in $2 G y$ per fraction schedule; followed by boost to the tumour volume alone with $14 \mathrm{~Gy}$ in $2 \mathrm{~Gy}$ per fraction. This modification allowed the restriction of dose to the critical structures, and good tolerance to dose escalation, thereby minimizing the acute and late sequalae of radiation.

After the treatment, $85 \%$ of the cohort achieved complete local response compared to $62.2 \%$ in the JCOG 9906 trial; one patient had residual disease and 2 patients progressed within the first 3 months after completion of treatment. 
The observed result was better than the retrospective Japanese trial [13] which compared high dose and standard dose radiation with concurrent chemotherapy, where the local control rate in high dose arm was 53\%. The disease-free survival rate at 2 year was $30.6 \%$ as compared to $47 \%$ in the Japanese trial.

With regards to late toxicity, the literature review showed that, the chance of radiation pneumonitis with dose escalation was about $23 \%$ compared to standard dose, which was $18 \%$. In the present study, no patient developed trachea-oesophageal fistula, pneumonitis, pleural effusion or pericarditis till the time of analysis. The percentage of patients that developed stricture was only $10 \%$ and they underwent SEMS placement following which they had better quality of life. This observation was close to the observed $10.4 \%$ chance of stenosis in JCOG trial with high dose radiation, with no significance when compared to standard dose radiation (8.2\%). The reduction in the CTV volume in the initial phase of irradiation may have been the reason for overall less toxicity [4].

In the present study it was also evaluated the association between the grade of dysphagia and the tumor volume, which not statistically significant $(p=0.157)$. The quality of life was evaluated at baseline and compared to 3 months post treatment. There was improvement in $85 \%$ of the patients, consistent with the results of JCOG trial [4]. Among the patients who failed, most of them were distant metastases (70\%) compared to loco-regional with distant metastases population (30\%). The higher proportion of patients in the stage III may have contributed to this. The overall survival and 2-year survival rate for this cohort of patients was 28 months and $53.7 \%$, respectively, at the time of analysis.

This result was similar to the retrospective analysis by Suh et al, who compared high dose versus standard dose (<54Gy versus >54Gy) with concurrent chemo-radiation in stages II-III oesophageal cancer [7]. The median dose in the high dose arm of this study was 63Gy. In multivariate analysis, 60Gy or higher radiotherapy was a significant prognostic factor for improved loco-regional control, progression-free survival and overall survival. The Suh et al. study [7] also showed that, there was no significant difference in the frequencies of late radiation pneumonitis, posttreatment oesophageal stricture or treatmentrelated mortality between the two study groups.
The present analyses in our institute, has its limitations with respect to the sample size and short follow up period. No patient underwent transoesophageal ultrasonography, therefore accurate Tstaging was not possible in most patients. Response assessment was based only upon oesophageal endoscopic evaluation and CT scan was not done to rule out disease extension outside the lumen. The local control and DFS were based on the symptoms/clinical findings and endoscopic observation alone, which may have led to underestimation of tumour recurrence.

The message from this study analysis is that, radiation dose escalation with concurrent chemotherapy is a feasible option for patients with advanced oesophageal squamous cell carcinoma, who are either unfit or refuse for surgical intervention, in achieving good local control. The oral intake status and quality of life was also better in the study population.

The toxicity of dose escalation may be minimized by implementing a limited CTV margin to tumour, with careful three-dimensional conformal planning, respecting the organs at risk constraints. study limitations such as short follow up duration, single centre study, small number of patients. autours contributed in writing of review of literature and guiding to conduct the study.

\section{Conclusion}

This prospective study evaluated the local response, toxicity and survival following dose escalation radiation therapy with concurrent chemotherapy, in patients diagnosed with stage I-III, squamous cell oesophageal carcinoma, who were either unfit or refused surgical intervention.

It was found that a complete response rate of $85 \%$ at 3 months, following the completion of radiation. The acute toxicities like esophagitis or stenosis $(10 \%)$, fairly resolve by 3 months leading to better oral intake. The quality of life of the patients post treatment improved in $85 \%$ of the patients. A longer follow up may predict the actual rate of late sequalae of radiation.

\section{What this study adds to the existing knowledge?}

It was observed from the present study that the high radiation dose was relatively safe and is effective in achieving good local control. 
The implementation of external beam radiation therapy, with reduced treatment volume reduces the sequalae of radiation.

Adequately sized and well-planned randomized control trials comparing the standard radiotherapy of $50.4 / 54 \mathrm{~Gy}$ to higher doses administered by external beam radiotherapy with present day techniques are required.

Further analyses may to identify its role in definitive treatment of advanced oesophageal squamous cell carcinoma.

\section{Author's contribution}

Dr. Ashwini L., Dr. Pradeep Kumar Kn: Guided the main author to prepare the study and writing the review of literature. Dr. Pavithran K: Gave concurrent chemotherapy and helped in managing the toxicities, preparing the study materials. Dr.

Ragavendra H.: Helped in treatment planning, dose calculations and proper dose distribution to the tumor and helped in statistical issues in the study.

\section{Reference}

01. Globocan 2018 world fact sheet. Available from: [Article] [Crossref]

02. Nishimura $Y$, Mitsumori M, Hiraoka M, Koike R, Nakamatsu K, Kawamura $M$. A randomized phase II study of cisplatin/5-FU concurrent chemoradiotherapy for esophageal cancerShort-term infusion versus protracted infusion chemotherapy (KROSG0101/ JROSG021). Radiother Oncol. 2009;92(2)260-265.

doi: $10.1016 /$ j.radonc.2008.12.012

[Crossref]

03. Versteijne $E$, van Laarhoven $H W$, van Hooft JE, van Os RM, Geijsen ED, van Berge Henegouwen MI, et al. Definitive chemoradiation for patients with inoperable and/or unresectable esophageal cancer- locoregional recurrence pattern. Dis Esophagus. 2015;28(5)453-459.

doi: 10.1111/dote.12215 [Crossref]

04. Kato K, Muro K, Minashi K, Ohtsu A, Ishikura S, Boku $N$, et al. Phase II study of chemoradiotherapy with 5-fluorouracil and cisplatin for Stage II-III esophageal squamous cell carcinoma- JCOG trial (JCOG 9906). Int J Radiat Oncol Biol Phys. 2011;81(3)684-690.

doi: $\quad 10.1016 / j . j$ ijobp.2010.06.033 [Crossref]
05. NCCN Clinical Practice Guidelines in Oncology. Esophageal and Esophagogastric Junction Cancers Version2. 2019.

Available from: [Article] [Crossref]

06. Minsky BD, Pajak TF, Ginsberg RJ, Pisansky TM, Martenson J, Komaki R, et al. INT 0123 (Radiation Therapy Oncology Group 94-05) phase III trial of combined-modality therapy for esophageal cancer- high-dose versus standarddose radiation therapy. J Clin Oncol. 2002;20(5)1167-1174.

doi: $\quad 10.1200 /$ JCO.2002.20.5.1167 [Crossref]

07. Suh YG, Lee IJ, Koom WS, Cha J, Lee JY, Kim $\mathrm{SK}$, et al. High-dose versus standard-dose radiotherapy with concurrent chemotherapy in stages II-III esophageal cancer. Jpn J Clin Oncol. 2014;44(6)534-540.

doi: $10.1093 /$ jjco/hyu047 [Crossref]

08. Kim HJ, Suh YG, Lee YC, Lee SK, Shin SK, Cho $B C$, et al. Dose-Response Relationship between Radiation Dose and Loco-regional Control in Patients with Stage II-III Esophageal Cancer Treated with Definitive Chemoradiotherapy. Cancer Res Treat. 2017;49(3)669-677.

doi: $10.4143 /$ crt.2016.354 [Crossref]

09. Fan CY, Su YF, Huang WY, Chao HL, Lin KT, Lin $\mathrm{CS}$. Definitive radiotherapy dose escalation with chemotherapy for treating non-metastatic oesophageal cancer. Sci Rep. 2018;8(1)12877. doi: 10.1038/s41598-018-31302-y [Crossref]

10. Song $T$, Liang $X$, Fang $M$, Wu S. High-dose versus conventional-dose irradiation in cisplatinbased definitive concurrent chemoradiotherapy for esophageal cancer- a systematic review and pooled analysis. Expert Rev Anticancer Ther. 2015;15(10)1157-1169.

[Crossref]

11. Teo MTW, McParland L, Appelt AL, Sebag-Montefiore D. Phase 2 Neoadjuvant Treatment Intensification Trials in Rectal Cancer- A Systematic Review. Int J Radiat Oncol Biol Phys. 2018;100(1)46-158.

doi: $10.1016 /$ j.ijrobp.2017.09.042 [Crossref] 
12. Zhang Z, Liao Z, Jin J, Ajani J, Chang JY, Jeter $M$, et al. Dose-response relationship in locoregional control for patients with stage II-III esophageal cancer treated with concurrent chemotherapy and radiotherapy. Int J Radiat Oncol Biol Phys. 2005;61(3)656-664.

doi: $10.1016 /$ j.ijrobp.2004.06.022 [Crossref]
13. Onozawa $M$, Nihei $K$, Ishikura $S$, Minashi $K$, Yano $T$, Muto $M$, et al. Elective nodal irradiation (ENI) in definitive chemoradiotherapy (CRT) for squamous cell carcinoma of the thoracic esophagus. Radiother Oncol. 2009;92(2)266269.

doi: 10.1016/j.radonc.2008.09.025 [Crossref] 